\title{
Las prácticas profesionales en la carrera de Relaciones Públicas
}

Gerardo Sanguine $^{(*)}$

\begin{abstract}
Resumen: En este artículo el autor reflexiona acerca de la importancia de la implementación de las prácticas profesionales en la carrera de Relaciones Públicas, las que brindan una experiencia de valor en la preparación de los futuros profesionales, así como un acercamiento a la realidad de la gestión que resulta el complemento ideal para la formación teórica. En este sentido, realiza un llamado a los profesionales y empresas del mercado a estar abiertos para ofrecer esta posibilidad a quienes se están formando, y ejemplifica con el caso de su propio programa de radio "Hablemos de Relaciones Públicas" de FM Palermo, que desde hace más de dos años ofrece un programa de prácticas extracurriculares a partir de la experiencia radial.
\end{abstract}

Palabras claves: Comunicación Corporativa - Comunicación Empresaria - comunicadores Dircom - educación superior - espacio joven en relaciones públicas - formación profesional - pasantías - prácticas profesionales - Relaciones Públicas.

[Resúmenes en inglés y portugués y currículum en las páginas 133-134]

El nivel de la formación de los alumnos de la carrera de Relaciones Públicas en las distintas universidades e institutos de nuestro país se ha superado mucho en los últimos años. Hay una mayor profundidad en el tratamiento de distintas materias, además se fueron incorporando los temas nuevos como -en su momento- el de la comunicación de crisis o más recientemente, el uso de las nuevas herramientas informáticas.

Es muy importante también que la carrera se dicte en universidades públicas y en las principales universidades privadas de nuestro país desde hace décadas. Pero se ha observado que hay un tema al que muchas casas de estudios deberían brindarle una mayor importancia, que es el tema de las prácticas extracurriculares.

El egresado pasará a ser un profesional completo cuando haya adquirido los conocimientos teóricos, indispensables para poder gestionar, junto a una experiencia que se adquiere con la práctica. Se puede decir que es un profesional completo cuando haya cumplido con estos dos requisitos, que en grado de importancia los consideraríamos por partes iguales. Planteado el tema de esta forma, se vuelve a destacar la necesidad de las prácticas extracurriculares en paralelo con la adquisición de los conocimientos teóricos.

Hay universidades que le dan el valor que esta cuestión merece, pero hay otras, en base a lo que se ha podido comprobar en forma directa, que no ocurre así y como se tratará de demostrar más adelante, es necesario que la academia impulse al alumno a esta práctica. $Y$ es un pecado que no se realicen. También en base a nuestra experiencia, hay una gran avidez por parte de los 
alumnos por conocer profesionales, por experimentar la práctica, por saber si realmente hay o no una relación estrecha entre lo teórico y lo práctico.

Una cosa es que desde la teoría se describa perfectamente una empresa o institución, y otra muy diferente cuando el alumno pueda recorrerla, hablar con su personal, vivenciarla. Las pasantías también son valiosas para la formación del estudiante, aunque en estos momentos las condiciones no son muy favorables para las empresas, lo que desalienta un poco su práctica. Es lógico pensar que el ofrecimiento de prácticas extracurriculares ha progresado a través del tiempo. Hace treinta años atrás, por ejemplo, eran casos muy aislados y dependían casi exclusivamente de la buena voluntad y certeza propia del profesor, que la visita con los alumnos a una empresa era indispensable para los estudiantes.

Para que estas prácticas se puedan materializar es también fundamental que haya una buena predisposición por parte de los que están ejerciendo la profesión en las distintas empresas, instituciones y consultoras, de recibir los alumnos y que los profesionales en actividad les dediquen un poco de su tiempo, que sin dudas luego redundará en mejores egresados, mejores candidatos para ocupar los puestos que se vayan produciendo en el mercado y una mejor reputación de la profesión que están ejerciendo.

Durante muchos años, se criticó desde las empresas y consultoras el nivel de los egresados de las distintas casas de estudios, pero muy poco se hizo para mejorar ese nivel. Las vacantes se cubrían con profesionales de otras disciplinas, periodismo y abogacía en su mayoría, pero felizmente esa práctica se fue perdiendo gracias a la mejora del nivel de las nuevas camadas de egresados. Tanto desde el ámbito académico, como desde el corporativo, se ha podido comprobar -y aquellos que tuvieron la oportunidad de recibir alumnos, también lo han podido apreciar- la avidez y dedicación que los alumnos ponen en esos trabajos prácticos, que además de toda la información que reciben, se suma la experiencia de la obtención y desarrollo de la entrevista dentro de la empresa.

Hoy podemos sentirnos orgullosos de que, cuando se publica un aviso para cubrir un puesto en una consultora o empresa, el principal requisito es que sea graduado en relaciones públicas.

\section{De la teoría a la práctica}

Tomando por ejemplo el caso de una gacetilla, hoy devenida en información para la prensa que los futuros relacionistas enviarán a los distintos medios de comunicación, como herramienta para que esa noticia impacte en los distintos públicos a los que quieren llegar.

Esa gacetilla que hasta algo más de quince años eran notas ensobradas que se entregaban a nombre del periodista en cada medio, con su correspondiente título, copete, desarrollo, cierre y los datos del emisor, a la recepción de hoy por mail, donde la persona que te la envía dice, hola, cómo estás? Te envío esta información por si puede ser de tu interés. Aunque aquí se sigue respetando el título gancho, el copete y el desarrollo, ha cambiado mucho la modalidad, que es mucho más directa y la reconocemos a esta forma como más ágil, amena y acorde con las nuevas herramientas que la tecnología nos ofrece.

En el aula, se puede explicar muchas veces detalladamente sobre su redacción, forma de envío y luego la revisión de su publicación en los distintos medios, pero en el segundo punto -que es su distribución- queda exclusivamente en manos de la práctica. Conocer los periodistas espe- 
cializados en cada medio, mantener un contacto fluido con ellos, que básicamente tiene que ver para cuando ellos necesitan alguna información sobre la empresa. Esa experiencia se adquiere con la práctica y además se necesita tiempo para que arroje los resultados esperados.

\section{Revisando la bibliografía}

Se puede afirmar por los libros revisados, que a través del tiempo son pocos los autores que se han dedicado a este tópico.

Por orden cronológico, comenzando por Fernández Escalante, que en el Capítulo XI "La actualidad y el futuro de las relaciones públicas", en el punto 11.2 denominado El Director de Relaciones Públicas, menciona:

Hay acuerdo general en que la persona que esté a cargo de esta función tiene que revestir las siguientes características (por orden de importancia): cultura general, grado universitario, conocimientos especializados de relaciones públicas, personalidad, contactos y amistades, tacto y condiciones diplomáticas, capacidad de redacción, cualidades de orador y conocimiento de idiomas, iniciativa y creatividad y la mayor práctica posible (1989, página 311 ).

Por su parte, Simon en el capítulo "Preparándose para una carrera en relaciones públicas", en una parte expresa:

Otras actividades valiosas fuera del programa de estudios incluyen el trabajo en la estación de radio o de televisión de la universidad, la participación en el gobierno estudiantil, y si es posible, un puesto en la sección local de la Public Relations Student Society of America (Sociedad de estudiantes en relaciones públicas de los Estados Unidos) (1996, página 476).

Finalmente Wilcox, que ha escrito su libro hace pocos años, ya menciona en un capítulo: "El valor de las prácticas internas" (2006, página 37), como él denomina a lo que nosotros estamos llamando prácticas extracurriculares y pasantías. Transcribo un párrafo donde justamente resalta el valor de las prácticas, las que considera una ventaja importante en el momento que el graduado quiera insertarse laboralmente. Dice así:

Las prácticas internas son extremadamente populares en el sector de la comunicación, y los estudiantes cuyos CV incluyen experiencia laboral práctica, junto con un buen expediente académico, disfrutan de una importante ventaja. La Comisión sobre Educación en Relaciones Públicas estadounidense considera que las prácticas internas son tan importantes que constituyen uno de las siete materias básicas que recomienda para cualquier programa de relaciones públicas de calidad de una universidad o un instituto de formación profesional. La práctica en una empresa es una situación en la que todo el mundo gana, tanto el estudiante como la organización. En la mayoría de los casos, el estudiante no solo recibe créditos académicos, sino que también obtiene un conocimiento de primera mano del trabajo que se realiza en el mundo profesional. Ellos ofrecen al alumno la ventaja para conseguir ese primer puesto de trabajo tan primordial tras su graduación. En muchos casos, el recién 
licenciado recibe un contrato de trabajo del empresario que le ofreció prácticas internas porque ya ha demostrado su valía.

También describe una serie de consultoras en Estados Unidos que tiene establecidos programas formales de prácticas laborales.

\section{Una experiencia radial}

Como corolario de la necesidad de la práctica extracurricular y tratando de ser coherente desde la docencia y la actividad en las empresas, se comentará una experiencia reciente, pero que ya lleva más de dos años de práctica, que es el establecimiento del espacio joven en un tramo de un programa radial temático sobre la profesión.

Se sabe que el conocimiento de la profesión está instalado a nivel empresarial; la concesión de las empresas nacionales en la década del noventa, trajo aparejada la designación de profesionales de la comunicación en los niveles que corresponden y con las funciones específicas por parte de las empresas que tomaron esas concesiones, empresas europeas y norteamericanas mayoritariamente, y luego la crisis del comienzo del dos mil, contribuyeron fuertemente a que el empresariado argentino comprenda la verdadera competencia de la profesión.

Falta que ese conocimiento baje al gran público, a aquel que no es de la profesión, que sabe cuál es la función de un médico o de un abogado, pero que no tiene una idea aproximada del quehacer de un relacionista público, y además tiene una imagen distorsionada en muchos casos por el mal uso que a través del tiempo se le dio y sigue dando a la denominación.

Ese es uno de los objetivos de este modesto espacio radial, que es llegar a la opinión pública para difundir qué hace un relacionista público, con la idea que en algún momento, le sea tan clara como lo que hace un profesional de otras disciplinas.

Se intenta alcanzar este objetivo, por un lado brindando una mirada radial sobre la profesión y contando lo que pasa en nuestro metier en las empresas, consultoras y ámbito académico, con la idea de llegar al gran público para que pueda formarse una real imagen de las actividades que desarrolla un profesional de las relaciones públicas.

El espacio joven -como su nombre lo indica- está destinado a los estudiantes y jóvenes profesionales y consiste en invitar a los estudiantes de la carrera para que visiten la radio, que puedan conocer cómo funciona un medio de comunicación social, con el que ellos van a tener una estrecha relación en su carrera profesional. Poder apreciar qué es lo que pasa con esa gacetilla, que ellos desde la empresa en la que trabajan enviaron a un medio radial.

Es una experiencia muy rica que cada día nos deslumbra con nuevas situaciones. Los visitantes tienen la posibilidad de decir sus primeras palabras al aire y hay desde aquellos que al comienzo se inhiben hasta los que llega el momento de decirles que se acaba el tiempo para su participación. Visitas en los que los estudiantes se pueden cruzar con profesionales de empresas, consultoras y del ámbito académico que concurren al programa para una entrevista, que a su vez ellos también pueden hacerles preguntas y lo más importante es que puedan comprobar que lo que los profesionales cuentan en sus entrevistas, que generalmente tiene que ver con la práctica de la profesión, esté en concordancia con lo que ellos están viendo en la teoría. Esto último les despierta un gran interés y les sirve también para ratificar su vocación por la carrera elegida. En 
casi todos los casos demuestran un gran atractivo por todos los temas que se tratan, ya sea tanto los referidos a las comunicaciones como a los temas de responsabilidad social empresaria. Además en cada intervención nos sorprenden con cosas nuevas, con la frescura y apasionamiento por descubrir más de esta atrapante actividad.

Como última prueba de grado de participación, se mencionará que dos estudiantes que en su momento visitaron el programa, han solicitado y hoy están colaborando en la producción y desarrollo del mismo en forma permanente y totalmente desinteresada, y es notable observar cómo se van perfeccionando en sus tareas a medida que transcurre su tiempo de participación.

\section{Conclusión}

Se considera a las prácticas profesionales en la carrera de relaciones públicas como una necesidad muy válida. Hay universidades que le dan una gran importancia al tema, hay una con un sistema de premios en base a la cantidad de visitas realizadas a medios, empresas y consultoras, otra que nos toca muy de cerca, que designa dos prácticas extracurriculares en el área de producción de nuestro programa radial, en forma rotativa durante todo el ciclo lectivo y desde el inicio del mismo, en agosto del 2007.

Y no solamente las prácticas extracurriculares y las pasantías son importantes, también lo es la participación de los alumnos en la Comisión de Estudiantes y Jóvenes Profesionales del Consejo Profesional de Relaciones Públicas de la República Argentina, que brinda esa posibilidad y que también coincide con lo expresado por Simon en su libro.

\section{Referencias Bibliográficas}

Fernández Escalante, F. (1989). Ciencia de la información y relaciones públicas. (3ra. Edición)

Buenos Aires: Macchi.

Simon, R. (1996). Relaciones públicas Teoría y práctica. (6ta. Reimpresión). México: Limusa. Wilcox, D. (2006). Relaciones Públicas. Estrategias y tácticas (8va. Edición). Madrid: Pearson

Educación S.A.

Summary: In this article the author reflects about the importance of the implementation of professional practices in the public relations career in order to offer a valued experience in the preparation of futures professionals, as well as an approach to the reality of the management that is the ideal complement for the theoretical formation. In this sense, the author invites the professionals and companies to be open to offer this possibility to those who are forming, and exemplifies with the case of its own radio program "We speak of Public Relations" of FM Palermo, that for more than two years had been offering complementary practices after the radial experience.

Key words: Communications director - Communication people - Corporate Communication - courses - professional formation - professional practice - Public relations - superior education - young space in public relations. 
Resumo: Neste artigo o autor reflexiona sobre a importância da implementação das prática profissionais na carreira de relações públicas, as cuais brindam uma experiência de valor na preparação dos futuros profissionais, assim como um acercamento à realidade da gestão que constitui o complemento ideal para a formação teórica. Neste sentido, faz um alerta aos profissionais e empresas do mercado a estar abertos para oferecer esta possibilidade a aquilos que estão-se formando. Além disso analisa o próprio programa de rádio "Falemos de Relações Públicas" de FM Palermo, que desde faz mais de dos anos oferece um programa de práticas extracurriculares a partir da experiência radial.

Palavras chave: Comunicação Corporativa - Comunicação Empresária - Comunicadores Dircom - educação superior - espaço jovem em relações públicas - estágios - formação profissional - práticas profissionais - Relações Públicas.

${ }^{*}$ ) Licenciado en Relaciones Públicas, egresado de la Universidad John F. Kennedy. [Ver CV completo en la página 155] 